\title{
No FOG = more clarity on self fertility
}

Many species go to great lengths to prevent or at least minimize mating between close relatives - or, in the case of plants, with themselves. Nematodes, on the other hand, show a blatant disregard for this taboo, as many species exist largely as selffertile hermaphrodites. Their existence, however, and our detailed genetic knowledge of one such worm, Caenorhabditis elegans, is a boon to those studying sex determination. But is it possible to describe a conserved pathway for hermaphroditism? By comparing the sex determination pathways of two sister species of Caenorhabditis, Sudhir Nayak and colleagues provide functional evidence that this is not the case, and that hermaphroditism has probably evolved by different genetic routes in the two species.

Thirty-one genes are known to be involved in establishing the germline and somatic sex of C. elegans. Although 30 of these genes have counterparts in the relative Caenorhabditis briggsae, the authors have found that one gene, fog-2, has no convincing orthologue in this species. This is puzzling given the fact that FOG-2 is needed to trigger spermatogenesis in the C. elegans hermaphrodite: here, a complex between FOG-2 and the RNA-binding protein GLD- 1 shuts down the synthesis of the feminizing TRA-2 protein, leading to sperm production. Given that C. briggsae hermaphrodites obviously produce sperm, and that this organism has presumed functional copies of all other known sex-determining genes, including $g l d-1$, how does this species establish the male-germ-cell fate?
RNA interference (RNAi) of the gld-1 transcript shows that this gene has opposite functions in the two Caenorhabditis species: whereas, as expected, knocking-down gld-1 in C. elegans hermaphrodites led to a feminized germline, RNAi masculinizes the germline of C. briggsae hermaphrodites, as sperm is produced at the expense of oocytes. The difference in gld-1 function between the two worm species is apparently limited to germline sex determination, as its requirement for oogenesis and meiotic progression has been conserved. However, this work does not tell us how GLD-1 might direct sperm production in C. briggsae in the absence of FOG-2 (and whether, in fact, it does), although the testing of some hypotheses is underway.

The lack of fog-2 in C. briggsae and the opposing roles of $g l d-1$ in germline sex determination indicate that hermaphroditism has evolved independently in these two species, and by extension in the whole Caenorhabditis clade. In fact, this work bolsters the existing view that sex determination mechanisms are incredibly labile, and depend more on a permissible anatomy and physiology (which is broadly similar across the clade) than on conserved genetic relationships.

Tanita Casci

(4) References and links ORIGINAL RESEARCH PAPER Nayak, S. et al. fog- 2 and the evolution of self-fertile hermaphroditism in Caenorhabditis. PLoS Biol. 3 e6 (2005)

FURTHER READING Zarkower, D. Establishing sexual dimorphism: conservation amidst diversity? Nature Rev. Genet. 2, 175-185 (2001) WEB SITE

Tim Schedl's laboratory:

http://www.genetics.wustl.edu/tslab/

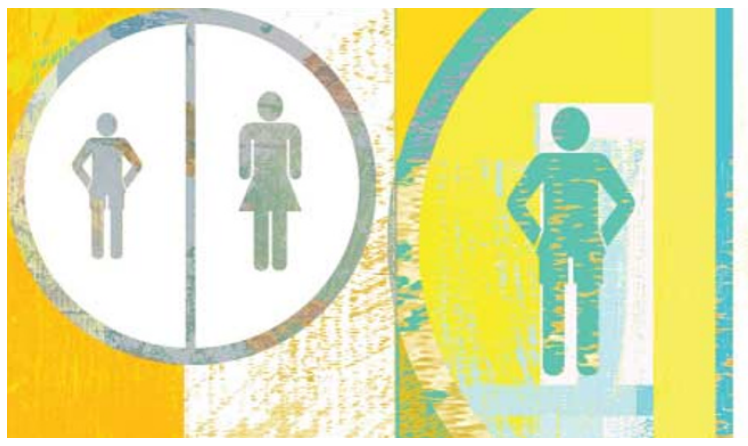

\section{IN BRIEF}

\section{STEM CELLS}

\section{p53 induces differentiation of mouse embryonic stem cells by suppressing Nanog expression.}

Lin, T. et al. Nature Cell Biol. 26 December 2004 (doi:10.1038/ncb1211)

Responding effectively to DNA damage is particularly important in embryonic stem (ES) cells. However, ES cells do not undergo efficient apoptosis or cell-cycle arrest in response to DNA damage. Lin and colleagues show that the tumour suppressor p53 triggers ES-cell differentiation by inhibiting expression of Nanog. This renders them susceptible to apoptosis and cell-cycle arrest, providing a mechanism to avoid the potentially disastrous consequences of DNA damage.

\section{SEX CHROMOSOME EVOLUTION}

A gradual process of recombination restriction in the evolutionary history of the sex chromosomes in dioecious plants.

Nicolas, M. et al. PLoS Biol. 3, e4 (2004)

Mammalian sex chromsomes are thought to have evolved through gradual loss of recombination between a pair of initially homologous autosomes, leaving a small pseudoautosomal region (PAR) that retains the ability to recombine. Nicolas et al. studied $\mathrm{X}$-linked genes in plants that use an $\mathrm{X}-\mathrm{Y}$ sex-determination system. Patterns of divergence of these genes from their Y-chromosome homologues indicated that sex chromosomes evolved through similar mechanisms in dioecious plants and mammals.

\section{HUMAN DISEASE GENETICS}

Loss-of-function mutation in tryptophan hydroxylase-2 identified in unipolar major depression.

Zhang, X. et al. Neuron 45, 11-16 (2005)

Decreased serotonin levels are implicated in several neuropsychiatric disorders, but the genetic basis of this is unclear. Zhang and colleagues identified a SNP in a gene required for serotonin synthesis - tryptophan hydroxylase 2 - resulting in a protein with reduced function. Among patients with unipolar major depression, 9 out of 87 carried this SNP, compared with 3 out of 219 in a control group, implicating defective serotonin synthesis as a major risk factor for unipolar depression and related conditions.

\section{MOLECULAR EVOLUTION}

The rate of DNA evolution: effects of body size and temperature on the molecular clock.

Gillooly, J. F. et al. Proc. Natl Acad. Sci. USA 102, 140-145 (2005)

The assumption that underlies the classical 'molecular clock' is that molecular evolution occurs at a universally constant rate. This study investigates the causes of the observed heterogeneity in the rate of molecular evolution. The authors' model, which is validated experimentally, indicates that the molecular clock ticks at different rates depending on metabolic status, and could explain rate variation among genes, taxa or temperatures. Importantly, this model supports the existence of a single molecular clock. 